\title{
ORIGINAL
}

\section{NECESIDADES DE ACCESIBILIDAD PARA ACCIONES PREVENTIVAS. UNA PERSPECTIVA DE LA POBLACIÓN EN MONTERREY, MÉXICO EN 2005}

\author{
María Eugenia Garza-Elizondo (1,2), Ana María Salinas-Martínez (1), Georgina Núñez-Rocha (1), \\ Enrique Villarreal-Ríos (1) y María Guadalupe Moreno-Monsiváis (2) \\ (1) Unidad de Investigación Epidemiológica y en Servicios de Salud. Instituto Mexicano del Seguro Social. \\ (2) Facultad de Enfermería. Universidad Autónoma de Nuevo León.
}

\section{RESUMEN}

Fundamento: Los programas integrados facilitan la tarea de concentrar acciones preventivas para grupos específicos. Sin embargo, los servicios de salud deben contar con la accesibilidad que es una condición básica para que la población acuda a ellos. El objetivo del trabajo fue identificar los municipios con mayor necesidad de accesibilidad de acciones preventivas utilizando información sintetizada en un índice de accesibilidad.

Métodos: Estudio transversal en ocho municipios de la zona metropolitana de Monterrey, México, a partir de una muestra aleatoria formada por 323 personas. Se consideró el uso de una acción preventiva por lo menos en el año previo a la fecha de la entrevista (vacunación, detección de diabetes o hipertensión). Se sintetizó la información en un índice de necesidades de accesibilidad considerando indicadores trazadores de uso y barreras por acceso, calidad del servicio y disponibilidad de recursos. Cada indicador fue estandarizado con base a puntajes normalizados $\mathrm{Z}$.

Resultados: El 98,9\% de la población estudiada recibió alguna acción preventiva. Los municipios con mayor necesidad de accesibilidad fueron Santa Catarina (Z -6,9), Apodaca ( $Z$-1,5) y Benito Juárez ( $Z$-1,2). San Pedro Garza García se vio más afectado por barreras de acceso geográfico o económico ( $Z$-3.5); Apodaca, por percepción de calidad $(Z-4,7)$; y Santa Catarina, por disponibilidad de recursos físicos, humanos o materiales $(Z-4,9)$.

Conclusiones: La mayor necesidad de accesibilidad se presentó en tres de los ocho municipios de la zona estudiada.

Palabras clave: Accesibilidad de los servicios sanitarios. Medicina Preventiva. Evaluación de necesidades.

Correspondencia:

$\mathrm{M}^{\mathrm{a}}$ Eugenia Garza-Elizondo

Verduzco 216 nte. Cd. Guadalupe NL, México

CP 67100

Correo electrónico: mariagarzae@intercable.net
ABSTRACT

\section{Accessibility Unmet Needs for Preventive Actions Seen from the Population's Perspective in Monterrey, México, during 2005}

Background: Integrated programs make the task of concentrating preventive actions for specific groups easier. However, health services must firstly be accessible, an essential condition for the population to use them. Hence, the objective of this study was to identify municipalities with the highest needs of accessibility of preventive actions through information synthesized in an accessibility index.

Methods: This cross-sectional study considered eight metropolitan municipalities of Monterrey, Mexico and 323 individuals sampled at random. We measured attendance for at least one preventive action the year before the survey, including vaccination, diabetes or hypertension diagnosis, and cervical or breast cancer among women. The accessibility index consisted of use and access barriers, quality and resource indicators standardized using Z-scores.

Results: Ninety-nine percent had attended health services for some preventive action. The municipality with the highest unmet need was Santa Catarina ( $\mathrm{Z}-6.9)$ followed by Apodaca ( $\mathrm{Z}$-1.5) and Benito Juárez (Z -1.2). San Pedro registered the highest unmet need concerning economical access barriers $(Z$ -3.5), whereas Apodaca was not good enough with quality perception ( $\mathrm{Z}-4.7)$ and Santa Catarina with perception of sufficient physical, human and material resources $(Z-4.9)$.

Conclusions: Three of the eight studied metropolitan municipalities registered the highest unmet need.

Key words: Health Services Accessibility. Preventive medicine. Needs Assessment. 


\section{INTRODUCCIÓN}

Uno de los grandes retos que se presentan en el ámbito mundial es el incremento de las enfermedades no transmisibles entre las que destacan las cardiovasculares, el cáncer y la diabetes ${ }^{1}$. En México la prevalencia de diabetes es del 7,5\% y de hipertensión del 30,7\%, mientras que el $60,5 \%$ de la población adulta de este país presenta factores de riesgo cardiovascular ${ }^{2}$. Es necesario garantizar que las acciones preventivas recomendadas estén al alcance de la población, de aquí los esfuerzos internacionales para tratar de reducir la inequidad en el acceso a través de políticas de promoción de prácticas preventivas con enfoque de riesgo ${ }^{1}$. Sumado a ello, el Sector Salud Mexicano ha implementado programas integrados de atención preventiva que faciliten las tareas de los trabajadores de la salud al concentrar lineamientos para acciones preventivas para grupos específicos de población, tomando en cuenta estándares establecidos previamente $^{3}$. Es importante mencionar que la población puede asistir a cualquiera de las unidades de salud de asistencia o seguridad social a realizarse acciones preventivas aunque no sea derechohabiente y sin costo alguno; y a las privadas, dependiendo de su capacidad de pago.

Dada su trascendencia, se debe investigar si se está satisfaciendo en la población la necesidad de acciones preventivas, lo que pudiera verse afectado por problemas de accesibilidad. La accesibilidad es una característica básica de los servicios de salud para que la población haga uso de las acciones preventivas ${ }^{4}$, ha sido definida como la relación entre las barreras de la búsqueda y la obtención de la atención ${ }^{5}$. De esta forma, en un extremo se encuentra la población en necesidad de la aplicación de acciones preventivas, mientras que en el opuesto está la disponibilidad de los recursos para tal fin; y entre ambos existen barreras que pueden entorpecer el uso de las mismas. Dichas barreras pueden ser de acceso geográfico, económico y organizacional, analizadas mediante el tiempo de traslado, pago del servicio, tiempo de espera, y la calidad del servicio percibida por el usuario ${ }^{6}$.

El estudio de accesibilidad integra entonces aspectos ampliamente abordados tanto en el ámbito internacional como en el nacional ${ }^{6-11}$. Datos de accesibilidad pueden encontrarse en informes estadísticos, plantillas de personal, cuadros fijos de material y medicamentos. Pero para su evaluación integral es importante tomar en cuenta la percepción de la población ya que al final será ella, quien logre vencer las barreras existentes para hacer uso de los servicios que se otorgan en las instituciones de salud. Tradicionalmente en la toma de decisiones o implementación de políticas de salud no se incluye el punto de vista del usuario de los servicios, sin embargo, es pertinente abordarlo para tratar de reducir las desigualdades de salud en las comunidades ${ }^{12}$.

Este estudio se distingue porque analiza la accesibilidad combinando en un índice aspectos de uso y barreras de acceso geográfico, económico y organizacional desde una perspectiva poblacional. Propone además, la jerarquización de zonas geográficas con mayor necesidad de mejorarla, lo que permitirá dirigir estrategias específicas hacia quienes más lo necesitan. Así el objetivo del estudio fue identificar los municipios en mayor necesidad de accesibilidad de acciones preventivas, mediante información sintetizada en un índice de accesibilidad.

\section{SUJETOS Y MÉTODOS}

Se realizó un estudio poblacional en población residente en la zona metropolitana de Monterrey, México, situada en la 
región nordeste del país, cuyo sector salud se conforma por instituciones de asistencia social (439 centros de consulta externa, 6 hospitales generales, 3 de hospitalización especializada, 1 centro de salud con hospitalización y 5 centros especializados), de seguridad social (75 unidades de consulta externa, 9 hospitales generales y 8 hospitales especializados); y privadas ${ }^{13}$. En todas ellas se desarrollan acciones preventivas de vacunación [doble viral (sarampión y rubéola), antineumocóccica, antiinfluenza o toxoide tetánico diftérico] o detección (diabetes e hipertensión; y en el caso de mujeres también cáncer cérvico uterino y mamario), entre otras; y para su cobertura es necesaria la participación del sector de salud mismo y de la población en general. Los requisitos de inclusión fueron tener 25 años o más de edad y contar con un mínimo de doce meses de residencia en alguno de los ocho municipios que conforman la citada zona metropolitana. Cabe mencionar que $83,8 \%$ de los habitantes del Estado de Nuevo León así como sus unidades de salud, se ubican en dicha área constituida por la capital del Estado Monterrey y siete municipios geográficamente colindantes ${ }^{13}$.

Se calculó un tamaño mínimo de muestra $(n=323)$ con base al supuesto "Habitantes de la zona metropolitana de Monterrey cuentan en promedio con un $70 \%$ de accesibilidad para uso de al menos una acción preventiva ${ }^{11}$, utilizando la fórmula para estimación de una proporción en donde el valor de $p=0,70$, el margen de error $d=0,05$ y el coeficiente de confidencia $C C=0,95$; el cual fue distribuido proporcionalmente de acuerdo al censo de habitantes de cada municipio ${ }^{13}$ de la siguiente manera: [Monterrey $\left(\mathrm{n}_{1}=112\right)$, Santa Catarina $\left(\mathrm{n}_{2}=23\right)$, San Nicolás de los Garza $\left(\mathrm{n}_{3}=50\right)$, San Pedro Garza García $\left(n_{4}=13\right)$, Escobedo $\left(n_{5}=23\right)$, Guadalupe $\left(n_{6}=67\right)$, Benito Juárez $\left(n_{7}=7\right)$ y Apodaca $\left(\mathrm{n}_{8}=28\right)$ ]. El proceso de selección se realizó por etapas en cada uno de los ocho municipios: 1) Elección aleatoria de un Área Geoestadística Básica (conjunto de manzanas edificadas y delimitadas por calles y avenidas $\left.{ }^{13}\right)$. 2) Identificación también al azar, de una manzana por cada 20 personas que se requerían para el estudio; este procedimiento fue basado en documentos de cartografía y realizado con apoyo del personal del Instituto Nacional de Estadística, Geografía e Informática (INEGI) del Estado de Nuevo León. 3) Una vez en la manzana, el encuestador con formación media superior y previamente capacitado incluyó la casa en la que se invitaría a participar a una persona. $\mathrm{Si}$ en el domicilio había más de una persona al momento de la encuesta, fue seleccionado al azar a quien participó en el estudio. En caso de no encontrarse ninguna persona en casa, se substituiría por otro hogar; situación que evitó repetir visitas a un mismo domicilio. El trabajo de campo se efectuó durante todos los días de la semana (incluyendo festivos) y en diferentes horarios.

Se diseñó un cuestionario que incluyó variables sociodemográficas y de uso. También sobre percepción de barreras para utilización y disponibilidad de recursos para acciones preventivas en unidades de salud de su propia comunidad, las cuales fueron colectadas desde la perspectiva de la persona mediante entrevista. Las opciones de respuesta estuvieron disponibles en escala dicotómica (sí, no), continua (gasto y tiempo de transporte, tiempo de espera) y de Lickert (muy poco, poco, regular, mucho). Se llevó a cabo una prueba piloto para evaluar la pertinencia del contenido del cuestionario y procedimientos diseñados para recolectar los datos; además, para verificar la comprensión, claridad y grado de aceptación de las preguntas.

Una vez reunida la información se procedió a la construcción del índice de accesibilidad con los siguientes indicadores: 
Uso: \% personas con alguna acción preventiva por lo menos en una ocasión durante el año anterior (el periodo de tiempo fue considerando el tiempo mínimo para realizarse cualquier detección). Los indicadores que a continuación se mencionan se refirieron a la última ocasión que se realizó alguna acción preventiva: Barreras por acceso geográfico y económico: \% personas que percibió $\leq 30$ minutos de tiempo de traslado; \% personas que percibió como poco y muy poco el tiempo de trayecto; $\%$ personas que gastaron un salario mínimo $(\$ 47,00$ pesos mexicanos) o menos en el traslado; \% personas que percibió como poco y muy poco el gasto de traslado. Barrera por calidad del servicio: \% personas que percibió un tiempo de espera $\leq 30$ minutos, desde que solicitó el servicio hasta que se le otorgó; $\%$ personas que percibió como poco y muy poco el tiempo de espera; \% personas que percibió como bueno y muy bueno el trato que recibió; \% personas que regresaría al mismo lugar para realizarse alguna acción preventiva. Disponibilidad de recursos: \% personas que percibió como suficiente el área física donde se realizó acciones preventivas; \% pacientes que percibió como suficiente el personal que realiza acciones preventivas; \% personas que percibió como suficientes los recursos para realizar acciones preventivas.

El plan de análisis consistió en frecuencias simples y determinación de la cantidad de población con necesidad de accesibilidad promediando los indicadores bajo estudio, en forma general y para cada uno de los ocho municipios. Posteriormente se sintetizaron las necesidades de accesibilidad en un índice considerando los 12 indicadores ya mencionados, previa estandarización con puntajes normalizados Z, de acuerdo con la siguiente fórmula:

$$
Z_{i}=\frac{X_{i}-\bar{X}}{S}
$$

donde $Z_{i}$ es el indicador (por ejemplo, percibió tardar $\leq 30$ ' de trayecto), $X_{i}$ es el valor observado del indicador (por ejemplo, \% percibió tardar $\leq 30$ ' de trayecto), $\bar{X}$ el promedio y $S$ la desviación estándar de los valores observados en todos los individuos (por ejemplo, $\bar{X}$ y $S$ de los cuatro indicadores del rubro barreras por acceso geográfico y económico). Se repite el proceso antes mencionado para cada municipio. En un segundo paso, el índice de necesidad de accesibilidad antes mencionado se estima sumando algebraicamente los puntajes $Z$ de cada indicador, siguiendo las siguientes fórmulas:

$Z$ del índice de necesidad de accesibilidad por barreras de acceso geográfico y económico $=$

$$
\begin{gathered}
Z_{1 \text { (percibió tardar 30'de trayecto) }}+Z_{2 \text { (percibió poco/muy poco }} \\
\text { tiempo de trayecto) } \\
+Z_{3 \text { (gastó hasta un salario mínimo en el traslado) }} \\
+Z_{4 \text { (percibió poco/muy poco gasto en trayecto) }}
\end{gathered}
$$

$Z$ del índice de necesidad de accesibilidad por barreras de calidad del servicio $=$

$$
\begin{aligned}
& Z_{1 \text { (percibió } \leq 30 \text { 'de espera) }}+Z_{2 \text { (percibió poco/muy poco tiempo }} \\
& \text { de espera) } \\
& +Z_{3 \text { (percibió trato recibido de bueno a muy bueno) }} \\
& +Z_{4 \text { (regresaría al mismo lugar a recibir acciones preventivas) }}
\end{aligned}
$$

$Z$ del índice de necesidad de accesibilidad por barreras de disponibilidad de recursos $=$

$$
\begin{gathered}
Z_{I \text { (percibió área física suficiente) }}+Z_{2 \text { (percibió personal suficiente) }} \\
+Z_{3 \text { (percibió material suficiente) }}
\end{gathered}
$$

$Z$ del índice general de necesidad de accesibilidad $=$

$$
\begin{aligned}
& Z_{1 \text { (uso) }}+Z_{2 \text { (percibió tardar } \leq 30 \text { ' de trayecto) }}+Z_{3 \text { (percibió }} \\
& \text { poco/muy poco tiempo de trayecto) }+Z_{4 \text { (gastó hasta un salario }}
\end{aligned}
$$

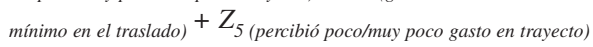

$$
\begin{aligned}
& +Z_{6(\text { percibió } \leq 30 \text { 'de espera) }}+Z_{7 \text { (percibió poco/muy poco tiempo de }} \\
& \text { espera) }+Z_{8 \text { (percibió trato recibido de bueno a muy bueno) }} \\
& +Z_{9} \text { (regresaría al mismo lugar a recibir acciones preventivas) } \\
& Z_{10 \text { (percibió área física suficiente) }}+Z_{11 \text { (percibió personal suficiente) }} \\
& +Z_{12 \text { (percibió material suficiente) }}
\end{aligned}
$$


Una vez obtenidos los resultados de la suma, éstos se ordenaron para jerarquizar las áreas geográficas en mayor necesidad. Los puntajes normalizados $Z$ fueron procesados con el programa estadístico EPIDAT@ versión $3.1^{14}$. Los puntajes pueden registrar signo positivo o negativo, un resultado negativo indica mayor necesidad. Así, surge un rango de necesidades de accesibilidad de acuerdo al signo y magnitud reflejando en un extremo, la no percepción de barreras para uso de acciones preventivas y, en consecuencia, la menor necesidad de accesibilidad. En el otro, la percepción de barreras y como resultado, la mayor necesidad de accesibilidad.

\section{RESULTADOS}

La tasa de respuesta fue del $100 \%$. La población encuestada presentó una edad promedio de 43,1 \pm 14,2 años; el 70,3\%, era de sexo femenino; $76,6 \%$ mencionó tener pareja; y $56 \%$ haber cursado estudios de secundaria y menos. Un $94,6 \%$ manifestó ser derechohabiente de alguna institución de seguridad social, siendo las unidades de salud del Instituto Mexicano del Seguro Social (IMSS) en donde el $58,4 \%$ de los encuestados, acudió a realizarse alguna acción preventiva. El análisis comparativo de características sociodemográficas entre la población blanco y la muestra señaló mayor porcentaje de personas con escolaridad secundaria en todos los municipios, el cual varió de 20,5\% a $79,1 \%$; en estado civil con pareja la diferencia estuvo entre $23,6 \%$ y $70,4 \%$ a excepción de Benito Juárez; con derechohabiencia, en San Pedro Garza García $(29,1 \%)$ y Apodaca $(21,9 \%)$; para sexo femenino, en Santa Catarina $(30,7 \%)$, San Nicolás de los Garza $(26,6 \%)$ y Guadalupe $(23,8 \%)$; y con ocupación hogar, en Santa Catarina $(23,9 \%)$, San Nicolás de los Garza $(41,8 \%)$ y Guadalupe $(35,9 \%)$ (tabla 1).

Tabla 1

Distribución porcentual de características sociodemográficas de la población de estudio (PE) y población blanco $(\mathrm{PB})^{\mathrm{a}}$ en municipios de Monterrey y su Zona Metropolitana

\begin{tabular}{|c|c|c|c|c|c|c|c|c|c|c|}
\hline & & \multirow{2}{*}{$\begin{array}{c}\text { Con } \\
\text { Seguridad } \\
\text { Social }\end{array}$} & \multirow{2}{*}{$\begin{array}{c}\text { Sexo } \\
\text { Femenino }\end{array}$} & \multirow{2}{*}{$\begin{array}{c}\text { Estado civil } \\
\text { con pareja }\end{array}$} & \multicolumn{4}{|c|}{ Escolaridad } & \multicolumn{2}{|c|}{ Ocupación } \\
\hline & & & & & Ninguna & Primaria & Secundaria & Profesional & Empleado & Hogar \\
\hline & & & & & & $\%$ & & & & \\
\hline \multirow[t]{2}{*}{ Monterrey } & PB & 69,8 & 51,4 & 27,3 & - & 48,8 & 32,4 & 18,8 & 53,6 & 25,5 \\
\hline & PE & 69,4 & 60,0 & $60,0^{*}$ & - & $15,2 *$ & $52,9 *$ & $29,2^{*}$ & $40,0^{*}$ & 32,9 \\
\hline \multirow[t]{2}{*}{ Santa Catarina } & PB & 76,2 & 50,5 & 36,2 & - & 55,2 & 26,4 & 9,4 & 57,1 & 26,1 \\
\hline & PE & 62,5 & $81,2 *$ & $68,8^{*}$ & - & $12,4 *$ & $68,8^{*}$ & 18,8 & 37,5 & $50,0^{*}$ \\
\hline \multirow{2}{*}{$\begin{array}{l}\text { San Nicolás de los } \\
\text { Garza }\end{array}$} & PB & 77,6 & 51,2 & 24,0 & - & 48,6 & 34,3 & 17,1 & 52,8 & 24,9 \\
\hline & PE & 72,2 & $77,8^{*}$ & $94,4^{*}$ & - & $16,7 *$ & $75,0^{*}$ & 8,3 & $30,6^{*}$ & $66,7^{*}$ \\
\hline \multirow{2}{*}{$\begin{array}{l}\text { San Pedro Garza } \\
\text { García }\end{array}$} & PB & 60,9 & 54,5 & 15,4 & - & 56,9 & 30,7 & 12,4 & 52,8 & 22,1 \\
\hline & PE & $90,0^{*}$ & 80,0 & $80,0^{*}$ & - & $10,0^{*}$ & $60,0^{*}$ & 30,0 & 60,0 & 40,0 \\
\hline \multirow[t]{2}{*}{ General Escobedo } & PB & 72,6 & 50,2 & 45,4 & 11,1 & 54,9 & 25,9 & 8,1 & 57,3 & 29,1 \\
\hline & PE & 80,0 & 53,3 & $73,3^{*}$ & - & $13,3 *$ & $86,7 *$ & - & 55,9 & 29,0 \\
\hline \multirow[t]{2}{*}{ Guadalupe } & PB & 73,0 & 51,0 & 31,3 & 6,9 & 50,3 & 29,5 & 13,3 & 54,5 & 26,2 \\
\hline & PE & 77,7 & $74,8^{*}$ & $85,4^{*}$ & 2,9 & $22,3 *$ & $61,2 *$ & 13,6 & $30,1^{*}$ & $62,1^{*}$ \\
\hline \multirow[t]{2}{*}{ Benito Juárez } & PB & 64,2 & 49,2 & 50,0 & 14,4 & 59,6 & 20,9 & 5,1 & 54,0 & 32,0 \\
\hline & PE & 22,2 & 66,7 & 66,7 & - & - & $100 *$ & - & 66,7 & 33,3 \\
\hline \multirow[t]{2}{*}{ Apodaca } & PB & 78,1 & 49,8 & 43,1 & 9,0 & 53,9 & 27,7 & 9,4 & 57,3 & 27,8 \\
\hline & PE & $100 *$ & 66,7 & $66,7^{*}$ & 5,6 & $33,3 *$ & $61,1^{*}$ & - & 55,6 & 33,3 \\
\hline
\end{tabular}

* $\mathrm{p}<0,05{ }^{\text {a }}$ Información del Sistema Municipal de Base de Datos, XII Censo General de Población y Vivienda, $2000^{21}$. 
Tabla 2

Perspectiva poblacional de Uso y Barreras para acciones preventivas* en Monterrey y su Zona Metropolitana

\begin{tabular}{|c|c|c|c|c|c|c|c|c|c|c|c|}
\hline & $\mathbf{Z M}^{* *}$ & 1 *** & $2 * *$ & $3 * *$ & $4 * *$ & $5 * *$ & $6^{* *}$ & $7 * *$ & $8^{* * *}$ \\
\hline \multicolumn{2}{|c|}{ Uso } & $\begin{array}{l}\text { - Usó por lo menos una Acción Preventiva* el } \\
\text { año anterior }\end{array}$ & 97,9 & 100,0 & 100,0 & 100,0 & 100,0 & 100,0 & 97,1 & 66,7 & 100,0 \\
\hline \multirow{8}{*}{$\begin{array}{l}\mathrm{B} \\
\mathrm{a} \\
\mathrm{r} \\
\mathrm{r} \\
\mathrm{e} \\
\mathrm{r} \\
\mathrm{a} \\
\mathrm{s}\end{array}$} & \multirow[t]{4}{*}{ Acceso } & - Percibió $\operatorname{tardar} \leq 30$ ' de trayecto & 88,4 & 87,1 & 75,0 & 86,1 & 70,0 & 100,0 & 91,3 & 100,0 & 88,9 \\
\hline & & - Percibió poco/muy poco tiempo de trayecto & 52,7 & 35,3 & 37,5 & 47,2 & 60,0 & 53,3 & 68,9 & 44,4 & 66,7 \\
\hline & & $\begin{array}{l}\text { - Percibió gastar hasta un salario mínimo } \\
\text { (\$47,00 pesos mexicanos) en trayecto }\end{array}$ & 91,8 & 97,2 & 50,0 & 88,9 & 55,6 & 100,0 & 87,2 & 87,5 & 100,0 \\
\hline & & - Percibió poco/muy poco gasto en trayecto & 49,0 & 43,1 & 100,0 & 48,1 & 44,4 & 61,5 & 59,6 & 25,0 & 50,0 \\
\hline & \multirow[t]{4}{*}{ Calidad } & $\begin{array}{l}\text { - Percibió } \leq 30 \text { ' de espera antes de recibir } \\
\text { acciones preventivas }\end{array}$ & 82,2 & 75,3 & 87,5 & 77,8 & 80,0 & 93,3 & 89,3 & 88,9 & 66,7 \\
\hline & & - Percibió poco/muy poco tiempo de espera & 47,6 & 29,4 & 43,8 & 69,4 & 40,0 & 66,7 & 58,3 & 22,2 & 33,3 \\
\hline & & - Percibió trato recibido de bueno a muy bueno & 97,3 & 97,6 & 93,8 & 97,2 & 100,0 & 100,0 & 97,1 & 100,0 & 94,1 \\
\hline & & $\begin{array}{l}\text { - Regresaría al mismo lugar a recibir acciones } \\
\text { preventivas }\end{array}$ & 92,5 & 94,1 & 93,8 & 97,2 & 100,0 & 100,0 & 88,3 & 88,9 & 88,9 \\
\hline \multirow{3}{*}{\multicolumn{2}{|c|}{$\begin{array}{l}\text { Disponibilidad } \\
\text { de Recursos }\end{array}$}} & $\begin{array}{l}\text { - Percibió área física suficiente para recibir } \\
\text { acciones preventivas }\end{array}$ & 96,6 & 97,6 & 31,3 & 94,4 & 90,0 & 100,0 & 98,1 & 100,0 & 100,0 \\
\hline & & $\begin{array}{l}\text { - Percibió personal suficiente para realizar } \\
\text { acciones preventivas }\end{array}$ & 90,8 & 96,5 & 75,0 & 91,7 & 90,0 & 80,0 & 89,3 & 100,0 & 88,9 \\
\hline & & $\begin{array}{l}\text { - Percibió material suficiente para realizar } \\
\text { acciones preventivas }\end{array}$ & 88,4 & 96,5 & 81,3 & 83,3 & 100,0 & 86,7 & 82,5 & 100,0 & 88,9 \\
\hline
\end{tabular}

* Vacunación [doble viral (sarampión y rubéola), antineumocóccica, antiinfluenza o toxoide tetánico diftérico] o detección (diabetes e hipertensión; y en el caso de mujeres también cáncer cérvico uterino y mamario). ** ZM = Zona Metropolitana $1=$ Monterrey $2=$ Santa Catarina $3=$ San Nicolás de los Garza 4 = San Pedro Garza García 5 = General Escobedo 6 = Guadalupe 7 = Benito Juárez 8 = Apodaca.

\section{Perspectiva poblacional de uso y barreras para acciones preventivas}

El 97,9\% de la población encuestada de la zona metropolitana expresó haber recibido alguna acción preventiva el último año. El 87,3\% no pagó por recibir una acción preventiva, quienes sí lo hicieron desembolsaron entre $\$ 10,00$ y $\$ 800,00$ pesos mexicanos $(\$ 16,23$ pesos mexicanos por $€$ ) considerado por el $97,5 \%$ como poco y muy poco. En lo referente al tiempo de trayecto, mencionaron haber tardado en llegar a la unidad de salud en promedio 20,4 $\pm 16,0$ minutos. El 63,1\% utilizó transporte público habiendo gastado en promedio $\$ 21,10 \pm \$$ 16,30 pesos mexicanos. El tiempo de espera promedio fue de 19,7 $\pm 15,2$ minutos.

El análisis de uso y barreras para acciones preventivas desde la perspectiva poblacional destacó que en la zona metropolitana el 49,0\% de los encuestados percibieron de poco a muy poco el gasto por trayecto y el $47,6 \%$, como poco a muy poco el tiempo de espera. En Monterrey, el 35,3\% y en Santa Catarina, el $37,5 \%$ percibieron como poco y muy poco el tiempo de trayecto En la tabla 2 se muestra a detalle el resto de los resultados. El porcentaje promedio de población con uso y barreras para uso en la zona metropolitana fue de $81,3 \pm 19,5$ siendo Santa Catarina, Benito Juárez y San Pedro Garza García las municipalidades con mayor población afectada (figura 1).

\section{Figura 1}

Porcentaje promedio e IC del $95 \%$ de población con uso y barreras de uso para acciones preventivas en la Zona Metropolitana de Monterrey, según municipio

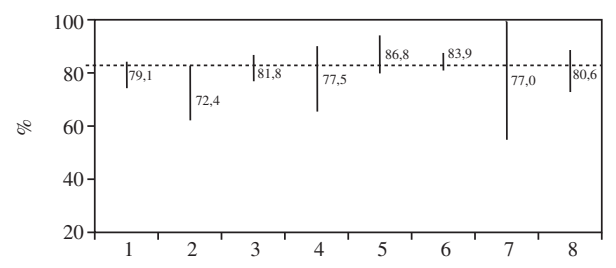

1 Monterrey. 2 Santa Catarina. 3 San Nicolás de los Garza. 4 San Pedro Garza García. 5 General Escobedo. 6 Guadalupe. 7 Benito Juárez. 8 Apodaca.

------ Porcentaje Promedio en la Zona Metropolitana. 
Figura 2

Índice general de necesidad de accesibilidad para acciones preventivas según percepción poblacional en Monterrey y su Zona Metropolitana

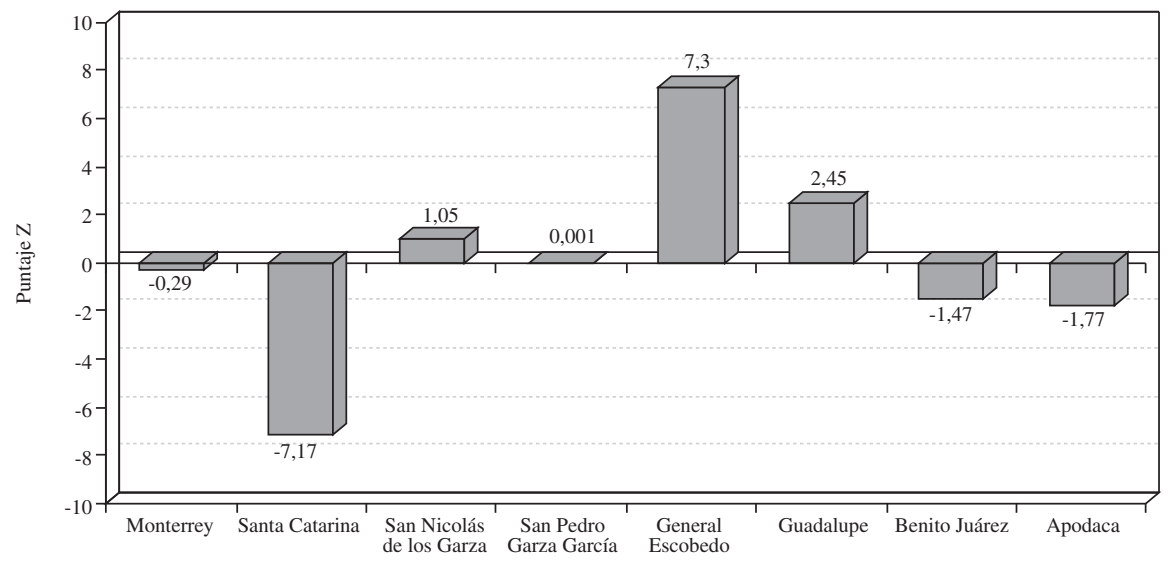

Figura 3

Índices específicos de necesidad de accesibilidad según perspectiva poblacional, por municipio
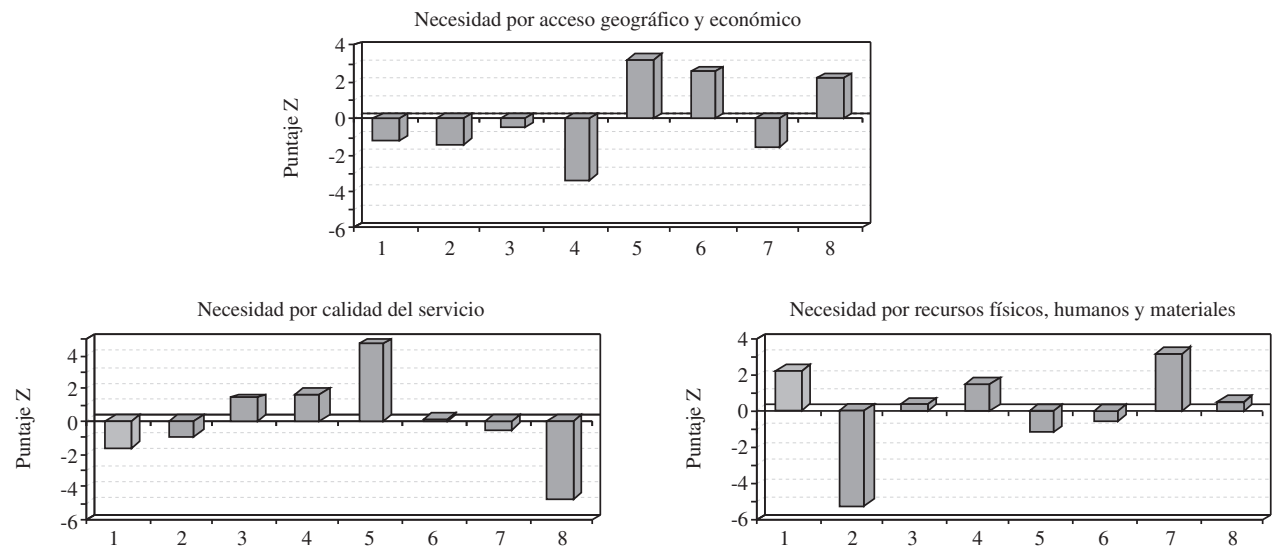

1 Monterrey. 2 Santa Catarina. 3 San Nicolás de los Garza. 4 San Pedro Garza García. 5 General Escobedo. 6 Guadalupe. 7 Benito Juárez. 8 Apodaca.

Índice de necesidad de accesibilidad para acciones preventivas en Monterrey y su zona metropolitana, según perspectiva poblacional

La información sintetizada de uso y percepción de barreras para uso y disponibilidad de recursos en un índice general de accesibilidad mostró a General Escobedo como el municipio con menor necesidad (puntaje $\mathrm{Z}$
7,3) mientras que Santa Catarina, aquél con mayor necesidad (puntaje Z -7,17) como se puede observar en la figura 2. Índices específicos revelaron que San Pedro Garza García tuvo la mayor necesidad percibida por barreras de acceso geográfico o económico (puntaje Z -3,5); Apodaca, por calidad del servicio (puntaje Z -4,7); y Santa Catarina, por disponibilidad de recursos físicos, humanos y materiales (puntaje Z -4,9) (Figura 3). 


\section{DISCUSIÓN}

Casi el 100\% de la población encuestada presentó el uso de alguna acción preventiva, contrario a lo reportado en el año 2004 para la misma zona, donde sólo un $53,5 \%$ usó acciones preventivas ${ }^{15}$. Esta diferencia pudiera ser resultado de la gran promoción que se ha dado a la atención preventiva integrada en las diferentes instituciones del sector salud durante los últimos años 1,16 .

El resultado del índice general de accesibilidad percibida mostró a Santa Catarina como la zona geográfica con mayor prioridad de satisfacer necesidades. El análisis detallado mostró que este municipio requiere mejorar en sus habitantes la percepción de disponibilidad de recursos llámense físicos, humanos y materiales si desea incrementar el uso de acciones preventivas. Se debe recordar que la interpretación que dan los individuos a su entorno se ve mediada por diversidad de estímulos. Así, la realidad puede ser transformada por malas experiencias con la falta de personal o material, lo cual afecta la percepción actual ${ }^{17}$. Lo anterior, debe ser estudiado posteriormente ya que no fue el objetivo del estudio. Por otra parte, Benito Juárez fue el área con mayor necesidad de satisfacer la accesibilidad por uso de acciones preventivas, situación que pudiera deberse a que éste se encuentra en pleno desarrollo y cuenta con extensiones territoriales de uso agrícola y otras que están en fase de convertirse en sectores habitacionales, dando como resultado una mancha urbana en expansión que requiere de campañas de comunicación del hábito de la prevención. También presenta porcentajes altos de población con barreras de acceso geográfico o económico. De hecho, llama la atención que mientras que la totalidad de la población encuestada percibió tardar menos de media hora para llegar a la unidad de salud a realizarse acciones preventivas, más de la mitad de la misma lo consideró mucho tiempo. Es importante mencionar que aunque las dos respuestas son desde la perspectiva de la población la primera de ellas en términos de tiempo absoluto se encuentra dentro de lo esperado por las instituciones de salud; mientras que la segunda incluye un juicio de las personas, en donde la idea que el tiempo ocupado en el trayecto a la unidad donde se realizarán la acción preventiva es más importante que la ganancia en salud por prevención ${ }^{18}$.

El municipio de San Pedro Garza García presentó el mayor número de población con barreras económicas, hacia el interior de éstas destaca que a poco más de la mitad se le hizo mucho el gasto de trayecto; contrario al $90 \%$ con barreras de acceso económicas por gasto de trayecto vencidas por el usuario en el Estado de Nuevo León, México ${ }^{11}$. Además, contrasta con el hecho de ser el municipio con más desarrollo económico en el estado de Nuevo León. Es importante destacar que la muestra de este municipio resultó sobre representada de individuos con seguridad social, por lo que las barreras de acceso percibidas en principio deben ser enfocadas hacia instituciones de seguridad social. El análisis de infraestructura médica de este municipio muestra que San Pedro Garza García cuenta con siete unidades de primer nivel [seis de la Secretaría de Salud (SSa) y solamente una del IMSS], además de un hospital de obstetricia e instituciones de salud privadas ${ }^{13}$. Esta situación hace que la población que tiene derecho a la seguridad social tenga que trasladarse a Santa Catarina o Monterrey para recibir acciones preventivas que trae como consecuencia mayor gasto de transporte. Aunque se sabe que la población puede asistir a la unidad de salud más cercana a su domicilio, ya que las acciones preventivas se pueden efectuar en las instituciones de seguridad o asistencia social sin importar condición de derechohabiencia (solo en las privadas dependerá de la capacidad de 
pago); pudiera estar prevaleciendo el traslado a aquélla de su preferencia no obstante ello implique mayor distancia, tiempo y gasto de transporte. Adicionalmente, la desinformación acerca de poder hacer uso de cualquier unidad pública del sector salud para efectuarse sus acciones preventivas, lo cual deberá estudiarse posteriormente.

Otro índice de necesidad de accesibilidad estudiado fue el de calidad del servicio. Las instituciones de salud deben garantizarla proporcionando un servicio oportuno, personalizado, humanizado, integral y continuo ${ }^{19,20}$. La zona geográfica con la máxima prioridad de mejorar calidad fue Apodaca, situación que se pudiera explicar por ser el $4^{\circ}$ municipio con mayor población y por lo tanto con mayor saturación de servicios con detrimento en indicadores como tiempo de espera y trato del personal ${ }^{21}$. Especialmente, las instituciones de salud de dicho municipio deben enfocarse a la percepción de tiempo prolongado de espera, a través de la promoción de estrategias que ofrezcan un ambiente confortable, respeto del orden de solicitud de la acción preventiva, abatimiento de la monotonía con difusión de temas de promoción de la salud ya sea con uso de medios electrónicos o impresos, e información del estatus del servicio (número de personas que anteceden, tiempo aproximado para recibir aplicación de acciones preventivas, etc.), entre otras ${ }^{18}$.

En cuanto a las limitaciones del estudio hay que tener en cuenta que la selección de la muestra fue al azar apegada al diseño muestral realizado con apoyo del personal del INEGI de Nuevo León. A pesar de que se acudió a los domicilios en diferentes horarios y días de la semana, se reconoce que los resultados están basados especialmente en población femenina, de escolaridad secundaria, con pareja, y dedicada al hogar. En el caso particular de los municipios de San Pedro Garza García y Apodaca predominaron encuestados derechohabientes por lo que se puede suponer que las respuestas proporcionadas estén representando a instituciones de seguridad social. Además, el tomar como fuente de información a la población implica subjetividad y se sabe que las apreciaciones se pueden ver influenciadas por experiencias anteriores en los servicios donde fueron efectuadas las acciones preventivas; sin embargo, es necesario que la percepción de las personas sea considerada en las evaluaciones de los servicios. Otra situación que puede estar marcando la tendencia de resultados es la memoria, es común que el uso de servicios se evalúe en los últimos 15 días ${ }^{6}$; en este trabajo se decidió interrogar acerca de éste durante el año anterior, por lo establecido respecto a la periodicidad de las detecciones y vacunas $^{3}$. Por lo que las percepciones a los indicadores explorados pudieran verse afectadas por el olvido o por el recordatorio a experiencias adquiridas en fechas recientes y no necesariamente ocurridas en la fecha de aplicación de la acción preventiva. Se sugiere considerar en estudios posteriores la verificación de la información en fuentes secundarias como cartillas de salud o expedientes clínicos.

Si bien, la mayor parte de los trabajos de accesibilidad se presentan con una medición objetiva, ya sea en el tiempo de trayecto, distancia o con la densidad de la población $1,2,8,9,22,23$ o reportan la calidad y satisfacción de los pacientes en forma independiente ${ }^{24,25}$. Existen también reportes de accesibilidad en forma integral que ponderan cada uno de sus indicadores ${ }^{11}$. Además, se ha estudiado en relación con mortalidad infantil, recursos humanos para la salud, estrato socioeconómico y afiliación en la seguridad social ${ }^{26,27}$. En este trabajo se hace una propuesta metodológica de evaluación de la necesidad de accesibilidad, mediante la creación de un índice desde la perspectiva del paciente con la enorme ventaja de 
determinar áreas geográficas o municipios en los que se tiene mayor necesidad, en este caso de accesibilidad. Se sugiere que en investigaciones posteriores se analice con necesidades de salud y estatus socioeconómico, para valorar si las acciones preventivas son empleadas por la población en ries$\mathrm{go}^{28}$. Estudios como el presente permiten a los directivos dirigir sus esfuerzos a aquellas zonas más desfavorecidas y así hacer eficientes los recursos destinados a la prevención de la salud.

\section{BIBLIOGRAFÍA}

1. Organización Panamericana de la Salud. CARMEN. Una iniciativa para Conjunto de Acciones para la Reducción Multifactorial de Enfermedades No transmisibles. Organización Panamericana de la Salud. 2003. Disponible en: http://www.paho.org/spanish/ad/dpc/nc/CARMEN-doc2.pdf (citado el 20 de marzo 2007).

2. Olaíz G, Rojas R, Barquera S, Shamah T, Aguilar C, Cravioto P, et al. Encuesta Nacional de Salud 2000. Tomo 2. La salud de los adultos. Cuernavaca, México: Instituto Nacional de Salud Pública; 2003.

3. Instituto Mexicano del Seguro Social. Programas Integrados de Salud. Guía Técnica 2002. México: Instituto Mexicano del Seguro Social; 2002.

4. Sousa MA, Villarreal E. Accesibilidad de los servicios de salud en población usuaria. Gac Med Méx 2000; 136: 213-219.

5. Frenk J. El concepto y la medición de la accesibilidad. Salud Pública Mex 1985; 27(5): 438-453.

6. Valdespino JL, Olaíz G, López-Barajas MP, Mendoza L, Palma O, Velásquez O, et al. Encuesta Nacional de Salud 2000. Tomo I. Vivienda, población y utilización de servicios de salud. Cuernavaca, México: Instituto Nacional de Salud Pública; 2003.

7. Gobina I, Vingre E. Evaluation of the youth friendly health services training programme. Youth friendly health services in primary health care in Latvia. 2005. Disponible en: http://www.papardeszieds.1v/public/down/YFHS_Final_Report.pdf (citado el 13 de septiembre de 2006).

8. Lostao L, Regidor E, Calle M, Navarro P, Domínguez V. Evolución de las diferencias socioeconó- micas en la utilización y accesibilidad de los servicios sanitarios en España entre 1987 y 1995/1997. Rev Esp Salud Pública 2001; 75: 115128.

9. Palomar R, Más C, Parra H, Rodríguez M. Accesibilidad a los dispositivos sanitarios de la Región de Murcia. Aten Prim 1996; 17: 507-511.

10. Seclén-Palacin J, Dorra Ch. Satisfacción de usuarios de los servicios de salud: Factores sociodemográficos y de accesibilidad asociados. Perú 2000. An Fac Med Lima 2005; 66: 127-141.

11. Villarreal E, González J, Salinas M, Garza E, Núñez G, Uribe M. Evaluación de la accesibilidad en los servicios de salud. Atención Primaria 2000; 25: 475-478.

12. Bedregal P, Quezada M, Torres M, Scharager J, García J. Necesidades de salud desde la perspectiva de los usuarios. Rev Med Chile 2002; 130: 1287-1294.

13. Instituto Nacional de Estadística, Geografía e Informática. Anuario Estadístico. Nuevo León. Edición 2001. México: Instituto Nacional de Estadística, Geografía e Informática; 2001.

14. Dirección Xeral de Saúde Pública, Xunta de Galicia, Organización Panamericana de la Salud, Instituto Superior de Ciencias Médicas de la Habana. EPIDAT 3.1. Análisis Epidemiológico de Datos Tabulados.

15. Garza ME, Villarreal E, Salinas AM, Núñez GM. Prácticas preventivas de los habitantes mayores de 25 años en Monterrey, México. Rev Esp Salud Pública 2004; 78(1): 95-105.

16. Jinés L, Martínez A, Medrano ME, Fernández I, Martínez G, Rendón E, Gutiérrez G. Atención Preventiva Integrada. Guía de las Actividades de Enfermería. México: Instituto Mexicano del Seguro Social; 2005.

17. Salazar J. La percepción, el conflicto y el estrés. Disponible en: http://www.gestiopolis.com (citado el 14 de agosto de 2006).

18. Vulcano G. (2007, 25 de febrero). El ser o parecer de los tiempos de espera. Capacitación en costos y gestión. Costs and taxes, Antequera, Duarte y Asoc. Bolivia. Disponible en: http://capacitacionencostos.blogia.com/2007/022507-el-ser-o-parecer-de-los-tiempos-de-espera.php ( citado el 25 de septiembre de 2007).

19. Aguirre H. Administración de la calidad de la atención médica. Rev Med IMSS. 1997; 35: 257-64. 
20. Roldán P, Vargas CR, Giraldo CP, Valencia GE, García CL, Salamanca LM. Evaluación de la calidad de la atención en salud. Un estudio de caso. Colombia Med 2001; 32: 14-18.

21. Instituto Nacional de Estadística, Geografía e Informática. Sistema Municipal de Base de Datos. XII Censo General de Población y Vivienda. 2000. Disponible en: http://sc.inegi.gob.mx/simbad/ index.jsp?c=125 (citado el 3 de marzo de 2007).

22. Fortney J, Cody M, Beck C. Geographic access and service use in a community-based sample of cognitively impaired elderly. J Appl Gerontol 2002; 21: 352-367.

23. McLafferty S, Grady S. Immigration and geographic access to prenatal clinics in Brooklyn, NY: A geographic information systems analysis. Am J Pubic Health 2005; 95: 638-640.

24. Banfield J. An evaluation of a new consultation approach/methodology to measuring residents' satisfaction and the findings. Oxford: Oxford City Council; 2006.

25. Jenkinson C, Coulter A, Bruster S, Richards N, Chandola T. Patient's experiences and satisfaction with health care: results of a questionnaire study of specific aspects of care. Qual Saf Health Care 2000; 11: 335-339.

26. Méndez-González RM. Cervera-Montejano MD. Análisis espacial de mortalidad infantil y servicios de salud en municipios indígenas de Yucatán, México, 1999-2000. Rev Biomed 2006; 17: 108117.

27. Lucuni Cuesta DI. Gómez Gutiérrez LF. Accesibilidad a los servicios de salud en la práctica de la citología reciente de cuello uterino en una zona urbana de Colombia. Rev Esp Salud Pública 2004; 78: 367-3777.

28. Hart JT. The inverse care law. Lancet 1971; 1 : 405-412. 